\title{
Update des Versorgungsvertrages nach § 132 i SGBV
}

\author{
Jürgen Koscielny ${ }^{1}$ Christoph Sucker ${ }^{2,3}$ \\ ${ }^{1}$ Charité Universitätsmedizin Berlin, Berlin, Germany \\ 2 Medizinisches Versorgungszentrum (MVZ) COAGUMED \\ Gerinnungszentrum Berlin, Berlin, Germany \\ 3 Medizinische Hochschule Brandenburg Theodor Fontane, \\ Brandenburg an der Havel, Germany \\ Hamostaseologie 2021;41:408.
}

Entsprechend des GSAV (Gesetz für mehr Sicherheit in der Arzneimittelversorgung) gemäß §130a Sozialgesetzbuch V (SGB V) erfolgte 2021 eine gesetzlich notwendige Aktualisierung der Preisstruktur durch den GKV-Spitzenverband mit den pharmazeutischen Unternehmen, die in den letzten Jahren Präparate zur Therapie der Hämophilie A neu auf den Markt gebracht hatten und bereits im AMNOG-Verfahren (Arzneimittelmarktneuordnungsgesetz) bewertet waren. Dies führt(e) zwangsläufig zu einer Aktualisierung der Rabattverträge der Krankenkassen im GKV-Bereich (gesetzliche Krankenversicherung) mit den pharmazeutischen Unternehmen (PU), die Medikamente im Versorgungsbereich der Hämophilie A auf dem deutschen Markt anbieten. Dies erfordert erstmalig die Aktualisierung eines Versorgungsvertrages nach $\S 132 \mathrm{i}$ SGB V, zuerst mit dem VDEK (Verband der Ersatzkassen e.V.). In Kurzform wird die wichtigste Neuerung dargestellt:

Es wird eine weitere Kategorie bzgl. der Wirtschaftlichkeit im Versorgungsbereich der Hämophilie A etabliert. Die Basispauschalen werden nicht reduziert, die Wirtschaftlichkeitspauschale wurde entsprechend der weiteren Kategorie angehoben und ergänzt. Diese kann durch eine zeitlich begrenzte Begleitungspauschale sogar noch weiter gesteigert werden. Die Anpassung erfolgt ersatzkassenindividuell. Die prozentuale Quotierung von mindestens 75\% („grüne Präparate“) zur Berechnung der Wirtschaftlichkeitspauschale(n) entfällt und vereinfacht die Vergütung. Es besteht damit zukünftig die Möglichkeit, eine höhere Vergütung durch den aktualisierten Versorgungsvertrag nach § 132 i SGBV vom VDEK zu
Address for correspondence PD Dr. med. Christoph Sucker, COAGUMED Gerinnungszentrum GmbH, Medizinisches Versorgungszentrum (MVZ), Tauentzienstraße 7b/c, 10789 Berlin, Germany (e-mail: cs@coagumed.de).

erreichen. Die Vorstände des BDDH und der GTH haben diesem Vorgehen zugestimmt. Der VDEK wird auf ALLE Vertragspartner zeitnah zu kommen. Es wird vom VDEK angestrebt, ab dem 01.10.2021 den aktualisierten Versorgungsvertrag nach $\S 132$ i SGBV zu starten.

Andere Versorgungsverträge nach $\S 132$ i SGBV von anderen Krankenkassen aus dem GKV-Bereich sind derzeit (noch) nicht betroffen.

\section{Einladung zur Mitgliederversammlung des BDDH und öffentlichem Teil am 03.03.2022 (66. Jahrestagung der GTH in Leipzig)}

Im Namen des gesamten Vorstandes sind ALLE Mitglieder zur Mitgliederversammlung des BDDH e.V. am Donnerstag, den 03.03.2022, 08.30 Uhr - 09.00 Uhr (nicht-öffentlicher Teil) im Rahmen der Jahrestagung der Gesellschaft für Thromboseund Hämostaseforschung (GTH) in Leipzig eingeladen. Es ist derzeit eine Präsenzveranstaltung vorgesehen, eventuelle Änderungen aufgrund der pandemischen Situation werden ggf. noch im Verlauf mitgeteilt. Wie üblich, wird die Tagesordnungspunkte der Veranstaltung im Verlauf noch kommuniziert.

Für den Vorstand der Deutschen Hämostaseologen Priv.-Doz. Dr. med. Jürgen Koscielny, 1. Vorsitzender Priv.-Doz. Dr. med. Christoph Sucker, Beisitzer des Vorstandes (c) 2021. Thieme. All rights reserved. Georg Thieme Verlag KG,

Rüdigerstraße 14,

70469 Stuttgart, Germany
DOI https://doi.org/

10.1055/a-1370-0205.

ISSN 0720-9355. 